\title{
Representaciones sociales acerca del impacto medioambiental de las empresas termoeléctricas en la bahía de Quintero-Puchuncaví
}

\section{Social representations about the environmental impact of thermal companies in the bay of Quintero-Puchuncaví}

\author{
Claudia Carrasco Aguilar ${ }^{1}$ \\ Pamela Morales Bierschwale ${ }^{2}$ \\ Jenniffer Salazar Fuentes ${ }^{3}$ \\ Universidad de Playa Ancha, Valparaíso, Chile
}

(Rec: septiembre 2014 - Acept: mayo 2015)

\begin{abstract}
Resumen
En la bahía de Quintero-Puchuncaví se plantea la tensión entre el desarrollo económico y la preservación de los recursos naturales y la calidad de vida. Se realizó un estudio cualitativo que abordó las representaciones sociales sobre el impacto medioambiental en habitantes de la bahía de Quintero-Puchuncaví. Se realizaron dos grupos focales con trabajadores de una escuela e integrantes del movimiento de Comunidades por un Derecho a la Vida, aplicando un análisis de contenido que arrojó como principales resultados que en la zona primaría el desarrollo económico e industrial en perjuicio de los recursos naturales, la salud, la calidad de vida y los derechos humanos de los habitantes del sector. Existiendo además, la idea de una política de ocultamiento de la información y medidas asistencialistas que intentarían, de forma ineficaz, mitigar el daño provocado. Se reconoce una actitud desfavorable en ambos grupos hacia el impacto medioambiental generado por las empresas, pues consideran que esta problemática ha superado todo límite. Esto moviliza vías de acción a nivel microsocial, en el caso de profesores, y a nivel de localidad, en el caso del movimiento.
\end{abstract}

Palabras clave: representaciones sociales, termoeléctricas, impacto medioambiental.

\begin{abstract}
In the Bay of Quintero-Puchuncaví there is a tension between economic development, preservation of natural resources and quality of life. This research presents a qualitative study that aimed to answer the question: Which social representations do the residents of the Bay of Quintero-Puchuncaví have about the environmental impact in the area? In order to attempt this, two focus groups were performed. The first one was composed by school employees and the second one by members of the movement "Communities for the Right to Life". Subsequently a content analysis was developed. This yielded as a main result that the Social Representation of these groups would be oriented by their own common sense, assuming as a part of it that in the area would prevail industrial economic development to the detriment of natural resources, health, quality of life and human rights of the inhabitants of the sector. It was also detected in these groups, the idea of an existing policy to conceal information from them, as well as welfarist measures that try ineffectively to mitigate the damage caused. Thus, it is recognized an unfavorable attitude in both groups to the environmental impact generated by industries, believing that this issue has exceeded all the limits. This mobilizes toward micro level actions in the case of teachers and local level actions in the case of the Movement.
\end{abstract}

Keywords: social representations, thermoelectric companies, environmental impact, inhabitants.

\footnotetext{
1 Correspondencia a: Claudia Lorena Carrasco Aguilar. Universidad de Playa Ancha, Departamento de Psicología, Av. Playa Ancha \#850, Playa Ancha, Valparaíso, Chile. Tel.: (56 3) 2220 5000. E-mail: claudia.carrasco@upla.cl

2 E-mail: pmoralesbierschwale@gmail.com.

3 E-mail: jenniffer.salazar.cf@gmail.com.
} 


\section{Introducción}

Los conflictos entre el desarrollo económico y la conservación de la biodiversidad han sido ampliamente reportados en la literatura; sin embargo, generalmente, han sido ignorados en el diseño de políticas públicas (Mills \& Waite, 2009, como se citó en Cárcamo, Cortés, Ortega, Squeo \& Gaymer, 2011). Se ha propuesto que estos conflictos pueden ser resueltos a través del progreso tecnológico. No obstante, en estos casos, las mejoras que apuntan a la eficiencia productiva o a la disminución de emisiones, están asociadas principalmente a metas macroeconómicas, y es poco probable que se desarrollen tecnologías de bajo impacto que aseguren la salud de los ecosistemas (Cárcamo et. al, 2011).

En este contexto, las políticas económicas no han sido afinadas para resolver directamente la crisis de la pérdida de la biodiversidad (Lawn, 2008, como se citó en Cárcamo et. al, 2011), por lo que las posibles soluciones debiesen estar localizadas en el contexto de políticas que reconozcan el conflicto.

Chile posee una economía diversificada, competitiva y abierta, orientada a los mercados externos y particularmente a los países desarrollados, y posee, además, una disponibilidad de fuentes de energía dadas sus características geográficas y climáticas (Programa Chile Sustentable, 2004). Según el "Informe país del estado del medio ambiente en Chile", en términos de desarrollo macroeconómico, esta modalidad ha resultado exitosa y dinámica, con una tasa de crecimiento estable, lo que ha provocado un incremento en la inversión por parte de empresas extranjeras (Centro de Análisis de Políticas Públicas, 2010). Sin embargo, esta modalidad de desarrollo adoptada por el país, ha privilegiado el crecimiento económico provocando un deterioro continuo del patrimonio natural, una alta dependencia de los mercados externos, un aumento de las desigualdades económicas y sociales y una mayor demanda energética que a la postre genera nuevos proyectos de generación de energía. Dadas estas características, continuando con el Informe país, ha faltado una estrategia más integral, que utilice la política de crecimiento económico en función de fines superiores de una estrategia de Estado, dejando a un lado el mejoramiento de la calidad de vida de los chilenos sobre la base del desarrollo ambiental sustentable (Centro de Análisis de Políticas Públicas, 2010).

Según Román y Hall (2011), en Chile existen cuatro sistemas eléctricos interconectados independientes: el Sistema Interconectado del Norte Grande (SING), que cubre el territorio comprendido entre las ciudades de Arica y Antofagasta (6.2\%) del total nacional; el Sistema Interconectado Central (SIC), que se extiende en las localidades de Taltal a Chiloé (92.3\%) del total nacional; el Sistema de Aysén que distribuye a esa región y el Sistema de Magallanes, que abastece a la región austral.

En nuestro país la generación de energía eléctrica tiene dos fuentes principales: hidráulicas y térmicas. En ambas, participan empresas eléctricas propietarias de centrales generadoras de capital privado, reguladas y fiscalizadas por el Estado. Las centrales termoeléctricas, a diferencia de las centrales hidroeléctricas, emplean combustibles fósiles como carbón, gas natural, petróleo y leña, para generar electricidad. Estas centrales emplean vapor de agua a alta presión producido por la combustión, para impulsar los generadores de electricidad. La ventaja de este sistema sobre las centrales hidroeléctricas es que, existiendo seguridad en el abastecimiento del combustible, la generación de energía eléctrica está asegurada (Superintendencia del Medio Ambiente, 2014). Las centrales Termoeléctricas a carbón, a diferencia de otro tipo de centrales termoeléctricas, se ubican en las orillas del mar, ya que requieren abastecerse de carbón el cual se transporta en la mayoría de los casos mediante vía marítima. Junto a esto, en su funcionamiento emplean grandes cantidades de agua de mar para su sistema de refrigeración, que también se emplean en muchos casos para eliminar el azufre que emana de la combustión del carbón. El agua empleada en el sistema de enfriamiento de las centrales a carbón es succionada a corta distancia de la costa, a una profundidad media de 7 metros, donde se origina la vida de los océanos (Contreras, Ibarra \& Ulloa, 2011), lo que se vincula con los impactos generados en la zona a partir de diversas formas de contaminación (González, Muena, Cisternas \& Neaman, 2008).

Debido a las implicancias en términos de calidad de vida que la instalación de estas centrales ha generado en la sociedad chilena, paulatinamente, ha comenzado un fuerte rechazo social a la instalación de termoeléctricas. Ello debido a la gran cantidad de proyectos de generación de electricidad que se han estado aprobando y que han resultado controvertidos, desencadenado múltiples conflictos sociales y descontento a nivel nacional acerca del tema energético (Román \& Hall, 2011).

En Chile no existe una legislación sobre las concentraciones máximas de elementos tóxicos permitidos en los suelos. A modo de suplir esta carencia, se utilizan legislaciones internacionales de referencia, lo que genera desajustes, puesto que en Chile lo que vale es el promedio de las emisiones del día, a diferencia 
de lo que ocurre en EE.UU. y países europeos, donde la norma se debe cumplir cada hora (Vega, Lyon \& Robles, 2011). Las normas de emisión de Chile se revisan cada cinco años.

En la Quinta región de Valparaíso, existen seis fuentes emisoras de contaminación sometidas a regulaciones, de las cuales las dos más importantes por la magnitud de sus emisiones, corresponden al Complejo Industrial Ventanas y la Fundición Chagres de Anglo American Chile (Centro de Análisis de Políticas Públicas, 2010), ubicadas en la bahía de QuinteroPuchuncaví. Por su lado, Puchuncaví, se sitúa como la comuna con los ingresos más bajos de la quinta región teniendo un $14,1 \%$ de población en condición de pobreza, mientras que Quintero posee un $18,1 \%$ de población en condición de pobreza (Instituto Nacional de Estadística [INE], 2010), lo que impacta en que la actividad industrial asociada a la presencia de estas empresas genere la producción de empleos, siendo significada como una oportunidad para la zona (De la Torre, 2010).

Esta situación se origina en la década de los sesenta, cuando comienzan a instalarse de manera progresiva distintas industrias en la comuna de Quintero, llegando en la actualidad a nueve: Codelco Chile; División Ventanas; Puerto Ventanas S.A.; AES Gener; Cemento Melón; Enap Refinerías; Gnl Quintero S.A; Gasmar; Comercial Catamutun S.A. y Oxiquim. Estas fueron emplazadas en la zona debido a su disponibilidad de agua y cercanía con los puertos de Quintero y Valparaíso ("El desafío medioambiental", 2011).

Esta situación ha llevado a que la calidad de vida de la población se vea afectada, pues estos gases han tenido consecuencias en el desarrollo socio-económico del sector, afectando la ganadería, agricultura y pesca. No obstante, fundamentalmente, ha tenido efectos nocivos para la salud, provocando una mayor incidencia de enfermedades pulmonares obstructivas crónicas y cardiovasculares; incrementando además, las infecciones y cáncer respiratorio, inflamación pulmonar y sistémica, exacerbación de los síntomas e incremento del asma, aumento de riesgo de infartos al miocardio, disfunciones vasculares, desarrollo de ateroesclerosis y malformaciones (Barría, 2009). Por otro lado, se encuentran vulnerados derechos establecidos en la Constitución Chilena como el derecho a la vida y a la integridad física y psíquica de las personas; así como el derecho a vivir en un medio libre de contaminación.

A tenor de esta realidad, se han llevado a cabo algunas investigaciones de tipo cualitativo que han llegado a concluir que muchos de los habitantes de las comunas mencionadas, actúan de forma pasiva frente a la contaminación y a la instalación de más empresas. Esto debido a que las ven como una fuente laboral importante, naturalizando las consecuencias devastadoras del progreso (De la Torre, 2010). En este sentido, Besnier (2010) analiza cómo las problemáticas ambientales provocadas por un modelo económico basado en la sobreexplotación, han provocado la exclusión de sus costumbres y su modo de vida y subsistencia, alterando la valoración de la identidad de pescadores artesanales de la zona.

A partir de lo anterior, cabe preguntarse por los discursos socialmente construidos por quienes, según estas investigaciones, naturalizarían la presencia de estas termoeléctricas, así como de quienes se encuentran organizados en la zona para resistir la presencia de las mismas, ambos grupos integrados por habitantes de la zona. De este modo, el foco de estudio se posiciona como un problema social, ya que es un fenómeno social con impacto negativo en las vidas de un segmento considerable de la población (Kohn, 1976).

La teoría de las representaciones sociales, permite operativizar los discursos socialmente construidos por estos grupos, llevando a construir la siguiente pregunta de investigación que busca ser respondida en el presente artículo: ¿Cuáles son las representaciones sociales sobre el impacto medioambiental en habitantes de la bahía de Quintero-Puchuncaví? De este modo, esta investigación buscó describir la información sobre el impacto medioambiental de las termoeléctricas manejada por los habitantes pertenecientes a la bahía de Quintero-Puchuncaví; describir la actitud sobre el impacto medio ambiental de las termoeléctricas de los habitantes pertenecientes a la bahía de QuinteroPuchuncaví y describir el campo de representación sobre el impacto medio ambiental de las termoeléctricas de los habitantes pertenecientes a la bahía de Quintero-Puchuncaví.

\section{Marco Teórico}

Con el fin de desarrollar una discusión conceptual y teórica que permita la profundización de los resultados, se presentan tres líneas conceptuales a seguir: Psicología Ambiental y Desarrollo Sostenible, Calidad de Vida y Derechos Humanos y la teoría de las Representaciones Sociales (RS en adelante). 


\section{Psicología Ambiental y Desarrollo Sostenible}

Según Wiesenfeld (2003), la psicología ambiental tiene aproximadamente treinta años de edad, aunque es reciente el interés sistemático de esta disciplina por el tema del Desarrollo Sostenible. Según Bravo (2010), la psicología ambiental en sus inicios estuvo influenciada por asuntos vinculados con la arquitectura, en relación con urbanistas u otros profesionales del cuadro urbano, siendo de gran utilidad la teoría de campo de Lewin (como se citó en Bravo, 2010) que apuntaba a entender la conducta como una función de una situación que comprende las condiciones del individuo y las variables del ambiente.

Sin embargo, para Bravo (2010), el modelo de desarrollo sustentable fue el que le otorgó importancia a la disciplina, visibilizando la posible contribución a la generación de cambios sociales en materia de protección del medioambiente. Para Wiesenfeld (2003), los postulados que dieron origen a la psicología ambiental se plantearon del mismo modo que los postulados que dieron origen al modelo de desarrollo sustentable. Esto es, estuvieron vinculados en ambos casos con la comprensión y abordaje de la problemática humanoambiental: "(...) en aras de una relación armónica entre ambos componentes, entendidos como totalidad" (p. 254).

Para la autora, el modelo de desarrollo sostenible y el modelo de la psicología ambiental, experimentan profundas diferencias, vinculadas con el foco de interés de ambas disciplinas. El modelo de desarrollo sostenible, se focalizaría en la conservación de los recursos naturales, con el fin de preservar la vida en el planeta y de posibilitar la supervivencia de las sucesivas generaciones, incluyendo la dimensión ambiental, económica, social e institucional. Por su parte, la psicología ambiental habría surgido en la academia y su foco estaría en el tiempo presente, enfatizando los contextos y niveles de análisis locales y las dimensiones ambientales y psicosociales. Sin embargo, para Wiesenfeld (2003):

No obstante las diferencias interperspectivas y la diversidad intrapersectiva mencionadas, resulta natural suponer que la Psicología Ambiental es una de las ciencias humanas más afín al objetivo fundamental del Desarrollo Sustentable y que los planteamientos de ambos requieren reconocerse en sus semejanzas en función del bienestar de la humanidad. (p. 254)
Para Bravo (2010), ubicar los problemas medioambientales y la relación del hombre con la naturaleza dentro de las preocupaciones de la psicología requiere adoptar una mirada distinta a la tradicional. Para el autor:

Las orientaciones actuales en psicología social han insistido en mostrar que la realidad social no es independiente de lo que podamos pensar de ella (Ibáñez, 1994). Esta misma fórmula puede aplicarse para el caso de la sustentabilidad y podemos plantear: lo que es "en realidad" la naturaleza depende en gran medida de cómo la representamos, es decir, qué pensamos de ella y cómo la imaginamos. (p. 224)

Para Wiesenfeld (2003), la entrada de los psicólogos ambientales en el tema de la sostenibilidad se fundamenta en el convencimiento sobre "(...) la responsabilidad individual y colectiva en el desarrollo de acciones que atentan contra la sostenibilidad del planeta y en la pertinencia de su dominio de experticia para aminorar, e incluso revertir esa tendencia”. (p. 255)

\section{Calidad de Vida y Derechos Humanos}

Para Bangher (2005), la calidad de vida conduce al análisis de los estilos de vida, a los valores que cada sujeto elige y que dan sentido a sus decisiones. Por ello, la calidad de vida sería la calidad biográfica de una existencia única, y por constituir el sentido de la vida del individuo, exige del contexto una respetuosa consideración de sus valores y los de la comunidad que habita. Según Vera (2007), la calidad de vida posee un significado eminentemente subjetivo, estando asociado con la personalidad, “(...) con su bienestar y la satisfacción por la vida que lleva, y cuya evidencia está intrínsecamente relacionada a su propia experiencia, a su salud y a su grado de interacción social y ambiental" (p. 285).

Siguiendo a García (1999), la Organización Mundial de la Salud habría especificado las condiciones que posibilitan lo que él denomina como exigencias para una calidad de vida, destacando las siguientes: un entorno físico de calidad, que incluye calidad de vivienda, disponibilidad de transporte y comunicaciones, salubridad pública, así como un ecosistema ambiental que posibilite un desarrollo sostenible a largo plazo. Se incluye además en estas condiciones un acceso a experiencias y recursos del entorno físico y sociocultural. Para el autor, el derecho primario de 
todo ser humano es desarrollar su personalidad lo más plena e integralmente posible, dando cuenta entonces del derecho primario de calidad de vida.

Para García (1999), la Declaración Universal de los Derechos Humanos, proclamada en 1948 por la Asamblea General de la ONU, supuso un avance decisivo y sigue siendo la plataforma básica para avanzar en la universalización y profundización de estos derechos. Sin embargo, el autor reconoce que es posible que ningún Estado, de los 186 presentes en la ONU hasta 1999, se manifieste en contra de la Declaración Universal de los Derechos Humanos, pero lo cierto es que, en su inmensa mayoría y en todos los continentes, la violación de los derechos es continua.

Para el autor, los cambios científicos, tecnológicos, económicos, políticos, sociales y culturales que se han dado desde la segunda mitad del siglo XX hasta hoy, plantean nuevos retos y demandas en la profundización y extensión de los derechos humanos de la solidaridad: "Un desarrollismo incontrolado e inhumano está devastando los recursos naturales, esquilmando la herencia de una tierra habitable a la que tienen derecho las generaciones venideras" (García, 1999). Para este autor, las nuevas condiciones plantean nuevas exigencias para lograr niveles más altos de calidad de vida. Es así cómo se van cristalizando nuevos derechos, reivindicándose entonces el derecho a un desarrollo sostenido que permita preservar el medio ambiente natural y el patrimonio cultural de la humanidad.

\section{Teoría de las Representaciones Sociales}

La teoría de las RS de Serge Moscovici, ofrece un marco explicativo acerca de los comportamientos de las personas, trascendiendo el marco cultural y las estructuras sociales más amplias. Su abordaje posibilita entender la dinámica de las interacciones sociales y aclarar los determinantes de las prácticas sociales, pues la representación, el discurso y la práctica se generan mutuamente (Abric, 2001). Para este autor, las RS constituyen sistemas cognitivos en los que es posible reconocer la presencia de estereotipos, opiniones, creencias, valores y normas que suelen tener una orientación actitudinal positiva y negativa.

Según Mora (2002), Moscovici estudió cómo las personas construyen y son construidas por la realidad social. A partir de sus elaboraciones propuso esta teoría cuyo objeto de estudio fue el conocimiento del sentido común enfocado desde su producción en el plano social e intelectual y como forma de construcción social de la realidad. Para Moscovici (1979), la RS es definida como una modalidad particular del conocimiento, por medio de la cual, lo que se busca es captar diferentes formas de comportamientos y comunicación entre individuos, que finalmente permitan dar cuenta de un sentido de coherencia, a través de un corpus organizado de conocimientos que permita que se integren en un grupo o en una relación cotidiana de intercambios.

Tajfel (como se citó en Páez, 1987) propone que las representaciones sociales requieren responder a tres necesidades: clasificar y comprender acontecimientos complejos y dolorosos; justificar acciones planeadas o cometidas contra otros grupos; y diferenciar un grupo respecto a los demás existentes, en momentos en que pareciera desvanecerse esa distinción. Por su lado, Jodelet (1986), añade que:

(...) el concepto de representación social designa una forma de conocimiento específico, el saber de sentido común, cuyos contenidos manifiestan la operación de procesos generativos y funcionales socialmente caracterizados. Hace alusión a una forma de pensamiento social. (p. 473)

Según Araya (2004), las RS son una forma de conocimiento que alude a un proceso y a un contenido. En concreto, consisten en una forma particular de adquirir y comunicar conocimientos, a la vez que conforman una forma particular de conocimiento, que constituye un universo de creencias en el que se distinguen tres dimensiones: la actitud, la información y el campo de representación (Moscovici, 1979). Para Mora (2002), la información corresponde a la suma u organización de conocimientos con que cuenta un grupo acerca de un acontecimiento, hecho o fenómeno de naturaleza social. Por su lado, la actitud implicaría la orientación favorable o desfavorable en relación con el objeto de representación social. Finalmente, el campo de representación consistiría en la organización de contenido de la representación, visualizando sus propiedades cualitativas, integrando información y actitud.

Para Araya (2004), el campo de representación se organiza en torno al esquema o núcleo figurativo que es construido en el proceso de objetivación. Para este autor, el análisis de las RS debe concluir con un esquema figurativo de pensamiento, sintético, condensado, simple, concreto, formado con imágenes vívidas y claras. El núcleo o esquema figurativo, implicaría convertir las ideas abstractas en formas icónicas, con forma gráfica y coherente que captura la esencia del concepto, teoría o idea que se trate de objetivar. El papel de la teoría aparece pues, en la forma en que estos modelos 
de comprensión se organizan para explicar o comprender una realidad. De este modo, y tal como proponen Arancibia, Rodríguez, Fritis, Tenorio y Poblete (2013), las personas configuramos nuestras RS de acuerdo a las condiciones de vida, experiencias y particular cultura en la cual nos desarrollamos. Así, las RS darían cuenta de una suerte de sentido común que expresa el pensamiento social (Castillo \& Winkler, 2010; Aparicio \& Mazzitelli, 2010), y han sido utilizadas con anterioridad como forma de acceder al conocimiento sobre aspectos medioambientales (Calixto, 2008).

De todas las opciones metodológicas para el análisis de las RS, este estudio se basó esencialmente en la propuesta de la teoría del núcleo central (Abric, 2001), bajo la cual es posible dar cuenta del contenido, estructura y funciones de la representación social. De este modo, se describen los mecanismos psicosociales que dan cuenta de este contenido, pero además, se puede establecer la forma del sistema periférico que permite que se dé el contenido particular del anclaje en la realidad del momento, autorizando modulaciones individuales e interviniendo en el proceso de defensa y de transformación de las representaciones. De este modo, queda en evidencia la forma en que se aborda el sistema central y periférico del cual nos habla el autor, a través de la presentación de elementos ordenados jerárquicamente alrededor del núcleo (Abric, 2001).

\section{Método}

\section{Tipo de estudio}

Este estudio se llevó a cabo bajo las consideraciones del Paradigma Cualitativo en Investigación Social, el cual destaca la comprensión como objeto principal de su análisis (Pérez Serrano, 1994). El estudio tuvo un carácter descriptivo, con un alcance temporal transversal, observando el fenómeno en condiciones naturales, intentando analizarlo en un período corto de tiempo (Hernández, Fernández \& Baptista, 2010).

\section{Participantes}

Esta investigación contó con la participación de habitantes de la bahía de Quintero-Puchuncaví, los que además, se encontraban divididos en dos grandes grupos: quienes trabajan como docentes en la zona, todos pertenecientes a la misma escuela básica, y quienes se encuentran organizados en el Movimiento de Comunidades Por El Derecho A La Vida. Se eligió ambos grupos, bajo las sugerencias de Quintana (2006), por medio de un muestreo de casos homogéneos, el que busca describir algún subgrupo en profundidad, por lo que el autor la señala como la estrategia empleada para la conformación de grupos focales. Para la conformación de cada grupo se llevó a cabo un procedimiento muestral en dos etapas (Salamanca \& Martín Crespo, 2007a): muestreo por conveniencia o voluntarios y muestreo en avalancha, eligiendo en primer lugar los sujetos que en ambas categorías quisieran participar. De este modo, ellos se encargaron de la convocatoria del resto de los participantes. A continuación, se presenta un cuadro que resume las principales características de los sujetos participantes del estudio:

Tabla 1.

Grupo Focal con trabajadores de la escuela

\begin{tabular}{cccc}
\hline Sexo & $\begin{array}{c}\text { Tramo } \\
\text { etario }\end{array}$ & $\begin{array}{c}\text { Años viviendo } \\
\text { en la zona }\end{array}$ & Dedicación \\
\hline Masculino & 40-50 años & 30 años & Profesor \\
Masculino & 30-40 años & 7 años & Profesor \\
Femenino & $30-40$ años & Lugareña & Profesora \\
Femenino & $50-60$ años & Lugareña & Auxiliar \\
Masculino & $50-60$ años & Lugareño & Orientador \\
\hline
\end{tabular}

Tabla 2.

Grupo Focal con miembros del movimiento de Comunidades por un Derecho a la Vida

\begin{tabular}{|c|c|c|c|}
\hline Sexo & $\begin{array}{l}\text { Tramo } \\
\text { etario }\end{array}$ & $\begin{array}{l}\text { Años viviendo } \\
\text { en la zona }\end{array}$ & Dedicación \\
\hline Femenino & 30-40 años & 10 años & Profesora \\
\hline Femenino & 40-50 años & Lugareña & Profesora \\
\hline Masculino & $50-60$ años & 7 años & Fotógrafo \\
\hline Femenino & 40-50 años & 25 años & $\begin{array}{l}\text { Asistente } \\
\text { social. }\end{array}$ \\
\hline Masculino & 30-40 años & Lugareño & Pescador. \\
\hline
\end{tabular}

\section{Técnicas de Producción de Información}

Se llevaron a cabo dos grupos focales, siguiendo las recomendaciones de Canales (2006), para quien la focalización se vuelve el centro de análisis. De este modo, cada grupo estuvo focalizado en una experiencia vivida, 
entendida directamente como representación o comprensión que tiene el sujeto de lo que hizo, hace o hará, desde sus conexiones de motivación y orientación, hasta la definición de contextos. Esta experiencia, fue focalizada a través de las vivencias y acciones, la perspectiva observadora del actor, las racionalidades de la acción y el testimonio y narración del habla focal (Canales, 2006).

\section{Procedimiento}

En primer lugar, se identificaron los potenciales colaboradores para el estudio así como las estrategias para acceder al sitio (Salamanca \& Martín-Crespo, 2007b), analizando la viabilidad de formar un grupo focal con quienes conformaron el Consejo Ecológico. $\mathrm{Al}$ analizar esto, descubrimos que éste había derivado en el Movimiento de Comunidades por el Derecho a la Vida, contactando a uno de sus dirigentes para conocer al grupo. En relación con los docentes, solicitamos la colaboración del Departamento de Administración Educacional de la comuna de Puchuncaví, cuyo psicólogo se encargó de realizar los contactos necesarios con distintos trabajadores de una escuela de la zona. Ambos se transformaron en los actores clave de este estudio (Salamanca \& Martín-Crespo, 2007b). Sin embargo, y pese a que los datos se encontraban saturados, se intentó llevar a cabo un tercer grupo focal con el fin de triangular la información pensando en seleccionar lugareños y profesionales, lo que finalmente no se logró. Cabe señalar que en los grupos focales apareció como un elemento redundante la dificultad de organización de la gente del sector.

\section{Aspectos éticos}

Se tomó en consideración las recomendaciones de Tójar y Serrano (2000), llevando a cabo los siguientes principios éticos: Involucrar a los participantes a través de la solicitud de sus opiniones respecto del tema y de la relevancia del mismo. Se negoció con todos los participantes asuntos como lugar y horario. Hubo autorización expresa antes de realizar los grupos focales, lo que se materializó en consentimientos informados, aceptando la responsabilidad de mantener la confidencialidad. Los sujetos que participaron del estudio, firmaron el consentimiento en el cual se mencionaba la posibilidad de nombrar las organizaciones de las cuales formaban parte con el fin de divulgar los resultados, aunque sus nombres quedarían expresamente omitidos. En este sentido, se consideraron los artículos 6 y 9 del Código de Ética de Psicólogos de Chile, que guardan conexión con la responsabilidad social del psicólogo, así como con el respeto por los otros.

\section{Procedimiento de Análisis}

Se llevó a cabo un análisis de contenido, con un fin descriptivo e inferencial, utilizado como técnica de análisis cualitativo. Siguiendo las recomendaciones de Alvira, García e Ibáñez (2003), el análisis no se limitó al contenido manifiesto de los mensajes, extendiéndose a su contenido latente. De este modo, y continuando con los autores señalados, se llevó a cabo el procedimiento seleccionando las unidades de muestreo (transcripciones de los grupos focales), unidades de contexto (extractos textuales de las transcripciones), unidades de registro (códigos que dan cuenta de las unidades de contexto), hasta llegar a establecer un total de tres categorías (casilleros en los que se clasifican las unidades de registro). Las categorías dieron cuenta en su contenido del análisis desarrollado, mientras que su denominación, obedece a las dimensiones de las representaciones sociales. Dentro de los aspectos de rigor científico, estos resultados se guiaron por el criterio de credibilidad (Ruiz Olabuénaga, 1996) a través de la triangulación de métodos (técnicas de producción de información), datos y fuentes.

\section{Resultados}

A continuación, se presentan los resultados del análisis de contenido, a través de las categorías que dan cuenta de las dimensiones de las RS.

\section{La Información}

Este apartado presenta los conocimientos con que cuenta cada grupo respecto del objeto de estudio en términos de creencias y emociones.

Respecto de los elementos de la información y en términos de las explicaciones sobre la realidad, surgen los siguientes tipos de creencias y expectativas como RS vinculadas a los trabajadores de la escuela que participaron en el primer grupo focal: Explicaciones ligadas a la percepción de que existe un ocultamiento de la información por parte de las autoridades y empresas respecto del impacto medio ambiental provocado por las termoeléctricas, así como también la creencia de que prevalece el desarrollo e interés económico por sobre el bienestar de los habitantes del sector: 
“(...) creo que hay muchas cosas que están ocultas, hay demasiadas cosas que el común de la gente desconoce, muchas veces no se les ha informado, uno después se entera por terceros, eso es una falta de respeto para la población, prevalece la parte monetaria ante la parte industrial (...)"

Asimismo predomina en el discurso la idea de que la situación actual de la zona sólo empeorará a medida que pase el tiempo, ya que los intereses económicos estarían siendo priorizados en los canales de comunicación a los que podrían acceder los lugareños:

“(...) es un problema que no se va a detener, mientras estén involucrado los intereses económicos de unos, esta situación en unos años más simplemente se va a acentuar y cuando llegue al límite quizás ahí se haga algo (...)"

De este modo, la información en este grupo, aparece más bien como una denuncia a la desinformación que perciben, existiría sobre la población en general. Con ello estarían reconociendo implícitamente que ellos poseen ciertos niveles de conocimiento respecto de las implicancias negativas de la presencia de termoeléctricas en la comunidad.

Estos elementos se encuentran presentes también en el grupo del Movimiento de Comunidades por un Derecho a la Vida, donde el conocimiento con que cuentan respecto del objeto de estudio, se encuentra vinculado a explicaciones ligadas con la percepción de que existiría un abuso de poder de parte de las empresas situadas en el cordón industrial de la zona y que operarían bajo políticas asistencialistas para mitigar el daño provocado a los habitantes del sector:

“(...) hay un exceso de abuso de poder de las empresas, que tratan de comprar a las personas con algunos proyectos, pavimentando las calles, regalando ciertas cosas, pero en el fondo el problema que hay es muy grave y aquí deberían ser más honestos con la población y deberían decirle a la gente que este ya es un sector industrial, y no para y a la gente erradicarla del sector e indemnizarla por todo el daño que han ocasionado (...)"

De este modo y coincidentemente con el grupo focal anterior, existe la creencia de que existiría un ocultamiento de la información a los habitantes del sector por parte de las autoridades y empresas respecto del real impacto medioambiental provocado en la zona.
Asimismo, predomina la sensación de que la situación empeorara a lo largo del tiempo y que las industrias deberían indemnizar a las personas afectadas por el daño provocado:

“(...) esta situación la veo caótica y va a generar una lucha grande, yo creo que esto va a desaparecer, esta va a ser una bahía totalmente industrializada hasta Quintero me atrevería a decir (...)"

\section{La actitud}

Este apartado presenta la orientación favorable o desfavorable en relación con el objeto de representación.

En términos de la actitud, los trabajadores de la escuela que participaron en el primer grupo focal, tienen una orientación desfavorable ligada a una sensación de desesperanza asociada a una baja expectativa de que la situación medioambiental mejore:

“(...) yo soy bien realista y esta es una lucha contra un gigante... esta situación superó todos los límites y no va a volver a ser lo mismo, ya no es lo mismo ya no queda nada está todo contaminado (...)"

Adicionalmente, predomina en el discurso una sensación de nostalgia y aflicción ligado a un sentimiento de identificación y de pertenencia, así como también de pesimismo en relación con el devenir de la zona:

(...) yo soy pesimista en ese sentido, no creo que la situación mejore, creo que sólo va a empeorar (...)"

En el grupo del Movimiento de Comunidades por un Derecho a la Vida, la actitud en relación con el objeto de representación, tiene una orientación desfavorable ligada a una sensación de pesimismo por no poder hacer nada en términos sustanciales, en torno a la situación entre la comunidad y las empresas de la zona. Ésta es significada dicotómicamente:

“(...) a mí también me da rabia, impotencia de no poder hacer nada y ver que las autoridades comunales se venden (...)".

Asimismo predomina en el discurso una sensación de impotencia y frustración que a pesar de todo, y a diferencia del grupo de los trabajadores de la escuela, los motiva a promover acciones ciudadanas en defensa de los derechos constitucionales de los habitantes del sector. 
De este modo, este grupo se habría empoderado a partir de la sensación de impotencia y frustración, mientras que el grupo de trabajadores de la educación estaría más bien desesperanzado y visualizarían pocas alternativas de acción:

“(...) tenemos una impotencia y una rabia me dan ganas de poner una bomba y que todo explote (...)"

\section{El campo de representación}

Este apartado presenta la organización del contenido, expresado por la relación de la información (creencias y afectos) y la actitud.

En términos de la organización del contenido en los discursos del grupo de trabajadores de la escuela, expresado por la relación de la información y la actitud, surgen explicaciones ligadas a la percepción de que el modelo económico adoptado por Chile intenta replicar modelos ya obsoletos en países desarrollados:

“(...) en realidad que todo esta tan manipulado, hay cosas que pasan por la imitación de otros lugares, el tener una vida más cómoda, mayor libertad, mayor progreso son todos modelos traídos de afuera... y no tenemos nada que ver con EEUU o Europa... al llegar a Chile estos modelos ya están trasnochados (...)"

Prevalece en el discurso además, la sensación de vulnerabilidad de los derechos humanos y preocupación, disconformidad y desconfianza en relación con la información oficial, contrastada con la experiencia personal de los habitantes del sector:

“(...) se le quiere mostrar algo a la comunidad que no es real, para que la gente no se involucre, porque en realidad porque tal vez en nuestras manos no está la solución (...)"

Además de lo anterior, existe una percepción de que el progreso económico de la zona implica un impacto directo sobre la calidad de vida de los habitantes del sector:

“(...) es una situación peligrosa y preocupante, porque es la casa de uno, además que nadie te va a asegurar que no van a instalar más termoeléctricas u otro tipo de industrias (...)"
“(...) la zona de Quintero ha sido muy golpeada con elementos que atentan contra la salud pero aquí hay muchas cosas que la gente no sabe... creo que tienes que vivirlo para saberlo, porque muchos pueden cuestionar lo que nosotros estamos viviendo, pero yo te digo que es así (...)"

En términos de la organización del contenido del Movimiento de Comunidades por un Derecho a la Vida, surgen explicaciones ligadas a la percepción de que, al existir conflictos de intereses, resultaría imposible lograr acciones mancomunadas en torno a la problemática medio ambiental que afecta a los habitantes del sector:

“(...) Las autoridades comunales se venden por nada, se dan una vuelta carnero en un minuto están en contra, protestan con nosotros, queman ataúdes y al día siguiente se dan una vuelta carnero firman tratos y están a favor de las empresas (...)"

$\mathrm{Al}$ interior de este grupo, también existe la idea de que habitantes del sector e industrias no pueden estar emplazadas en el mismo lugar físico y es por este motivo que debiesen tomarse medidas radicales:

“(...) antes me causaba duda yo pensaba tal vez usar energías limpias, pensaba como vivir con estas industrias, pero no se puede, ellos se van o nos vamos nosotros (...)"

“(...) en ninguna parte del mundo han puesto esto que esta acá, la sinergia de termoeléctrica con fundición es un problema grave, las cuestiones más insólitas pasan aquí, además las normas son para algunos contaminantes porque no están todos $(\ldots) "$

Finalmente, vuelve a aparecer el tema de la organización colectiva en estos discursos, ya que prevalece una sensación de que la mejor forma de provocar cambios sustanciales, es organizando a la comunidad en torno a objetivos comunes:

“(...) las organizaciones deben unirse y enfrentar esto de verdad, para qué seguir poniendo escuelas de proyectos si no tienen sentido, falta mucho por hacer pero creo que por ahí va el camino (...)" 


\section{Discusiones}

El discurso de los grupos focales estructura y objetiva concepciones compartidas que se pueden evidenciar en un esquema figurativo de pensamiento, sintético, condensado, simple y concreto, formado con imágenes vividas y claras, donde las ideas abstractas se conviertan en formas icónicas. A esto se refieren los autores de las RS cuando hablan del núcleo o esquema figurativo (Araya, 2004).

A continuación se presenta un esquema que captura la esencia del concepto, teoría o idea que se busca objetivar. Esta simplificación en la imagen es lo que le permite a las personas conversar y también comprender de forma más sencilla las cosas, a los demás y a ellas mismas y a través de su uso, en diferentes circunstancias. De este modo se convierte en un hecho natural.

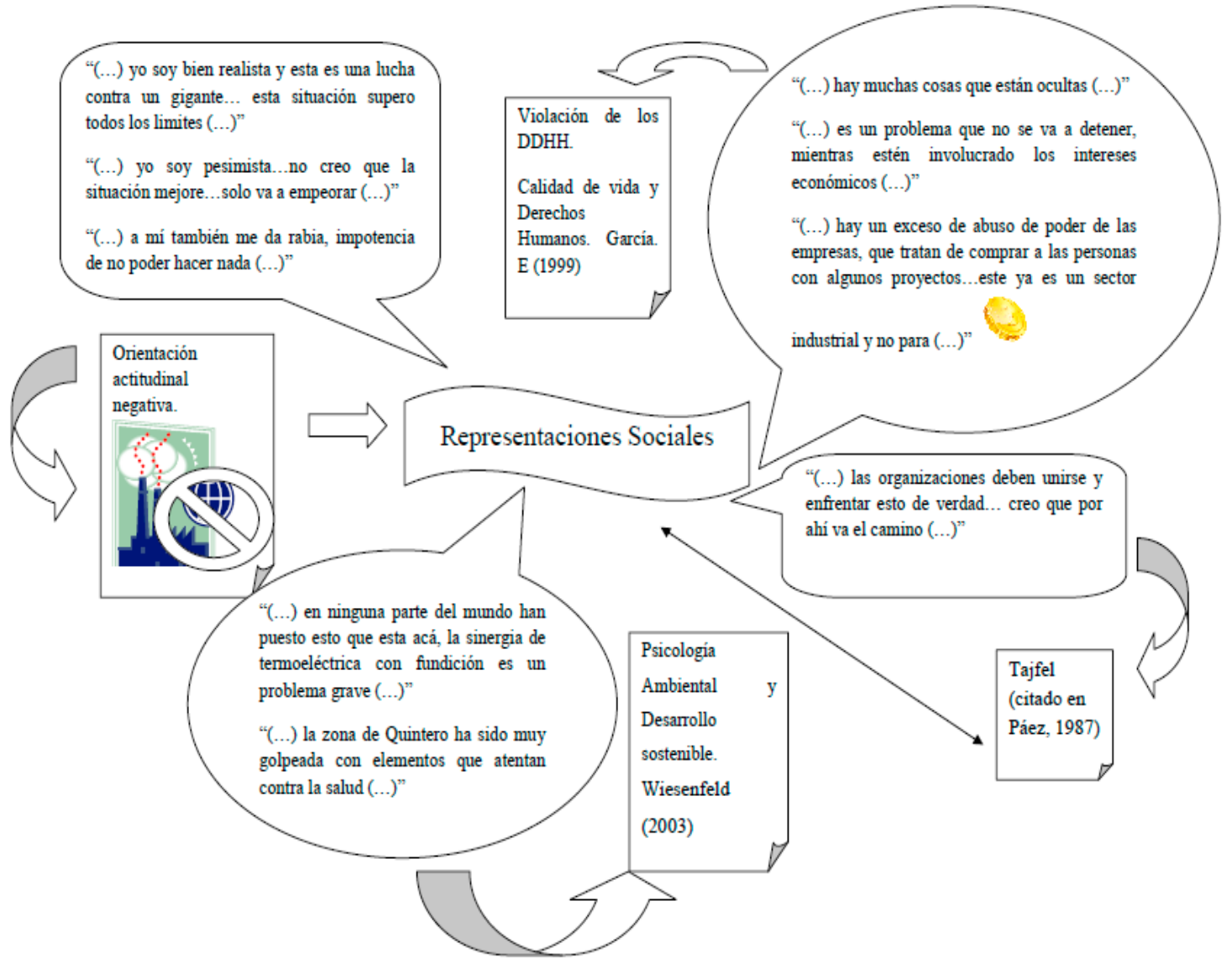

Figura 1. Núcleo figurativo de las RS.

$\mathrm{Al}$ establecer una relación en términos teóricos/ empíricos podemos distinguir los elementos expuestos a continuación.

A través de los diversos discursos de los participantes del estudio, se puede determinar que existiría en la zona una creciente actividad económica enmarcada dentro de una política de mercado que privilegiaría el desarrollo económico industrial en perjuicio de los recursos naturales. En este sentido se estaría utilizando una política de ocultamiento de la información y medidas asistencialistas que intentarían de forma ineficaz mitigar el daño provocado.

Esta situación afectaría directamente a la salud, la calidad de vida y los derechos humanos de los habitantes del sector, por estar siendo constantemente vulnerado el derecho primario que tiene todo ser humano a desarrollar su personalidad lo más plena e integralmente posible, teniendo acceso a experiencias y recursos del entorno físico sociocultural dentro de la comunidad que habita. Esto se vincula con los 
planteamientos propuestos por García (1999), ya que los aparentes cambios científicos, tecnológicos, económicos, políticos, sociales y culturales que estarían ocurriendo en Puchuncaví y Quintero desde la segunda mitad del siglo XX hasta hoy, plantearían nuevos retos y demandas en la profundización y extensión de los derechos humanos de la solidaridad, poniéndolos en tela de juicio. De este modo, y continuando con el autor, las RS de estos grupos estarían orientadas a asumir como parte de su propio sentido común, que el desarrollismo se estaría volviendo cada vez más incontrolado e inhumano, trayendo como consecuencia lo que advierte García (1999), es decir, la devastación de los recursos naturales.

De este modo, según el autor, se dificultaría el logro de justos niveles de calidad de vida. De este modo, se van cristalizando nuevos derechos, reivindicándose el derecho a un desarrollo sostenido que permita preservar el medio ambiente natural y el patrimonio cultural de la humanidad. Sin embargo, cabe la duda de si realmente los entrevistados en ambos grupos consideran en sus representaciones que esto es un derecho. Para el grupo de integrantes del Movimiento de Derechos por la Vida, efectivamente este es un derecho, lo que no sólo se evidencia en su nombre, sino también en lo que manifiestan al señalar que la frustración e impotencia los lleva a la organización.

De este modo, la organización se vincularía con la necesidad de hacer valer un derecho, mientras que los trabajadores de la escuela parecen naturalizar la idea de que los derechos humanos no contemplarían el derecho a vivir en un ambiente sano. Esto debido a que la impotencia en ellos, se convierte en desesperanza e inmovilidad. Como si no hubiese nada más que hacer y efectivamente la transformación de la situación de desvinculación de derecho dependiese de las empresas y autoridades.

Siguiendo la misma lógica, este desarrollo económico sostenido del cordón industrial de la zona, estaría representado socialmente como un espacio de privilegio sobre el progreso económico exponencial, sobreexplotando los recursos naturales no renovables, deteriorando el medio ambiente y obviando una política a la base de un modelo de desarrollo sustentable. Esto se vincularía con los planteamientos de Wiesenfeld (2003), para quien la responsabilidad debe ser individual y colectiva en el desarrollo de acciones para resistir aquellas que atentan contra la sostenibilidad del planeta y en la pertinencia de su dominio de experticia para aminorar, e incluso revertir esa tendencia. De este modo, las industrias estarían siendo representadas como impulsoras de prácticas que atentan contra la sostenibilidad del planeta.

En términos de la construcción intersubjetiva y de las dinámicas interaccionales que se construyen en torno a la problemática medio ambiental, es posible señalar que en los entrevistados, existe una tendencia a evaluar que sí existe impacto en las relaciones sociales de los habitantes de la Bahía, a partir de la instalación de las termoeléctricas en el sector. Asimismo, al analizar la experiencia concreta con las que se enfrentan a diario, queda en evidencia que la relación cotidiana con estas empresas va influyendo en la forma de ser de estos habitantes, condicionando tanto su identidad social como la forma en que perciben la realidad social.

Con base en lo anterior, es posible concluir que estas expresiones del pensamiento social, tendrían una orientación actitudinal negativa hacia el impacto mediambiental pues consideran que esta problemática ha superado todo límite. Este discurso posee relación con los planteamientos de Moscovici (1979), quien señala que las RS constituyen sistemas cognitivos en los que es posible reconocer la presencia de estereotipos, opiniones, creencias, valores y normas que suelen tener una orientación actitudinal positiva y negativa.

A raíz de lo anterior, ambos grupos asumen acciones distintas derivadas de actitudes similares, lo que se presenta como algo interesante a profundizar. Sin embargo, la aparente inmovilidad del grupo de trabajadores de la escuela no sería tal. En cuanto existirían algunas acciones de resistencia a nivel microsocial, construyendo algunas actividades en el colegio vinculadas con el cuidado del medioambiente a través de actividades curriculares como el Taller de Medioambiente. En cambio, los miembros del Movimiento por un Derecho a la Vida realizan acciones a nivel de localidad, con el propósito de informar, difundir y actuar con el mayor número de personas posible, con mayor conciencia también de la condición de derecho que estaría siendo vulnerada.

De lo anterior, es posible establecer una relación con los postulados de Tajfel (como se citó en Páez, 1987) quien propone que las RS requieren responder a tres necesidades: clasificar y comprender acontecimientos complejos y dolorosos; justificar acciones planeadas o cometidas contra otros grupos, y para diferenciar un grupo respecto a los demás existentes, en momentos en que pareciera desvanecerse esa distinción. El componente psicosocial ha tenido poca presencia en estudios sobre el medio ambiente y esto ha provocado poca precisión conceptual y una inconsistente formulación de estrategias de políticas públicas para su 
abordaje. Asimismo las relaciones entre los aspectos psicológicos, sociales y ambientales han tendido a conceptualizarse en términos sistémicos o ecológicos. Sin embargo, las temáticas medio ambientales, no pueden contemplarse al margen de los aspectos psicosociales y culturales.

Cabría preguntarse en futuras investigaciones respecto de aquello que lleva a que un grupo organice acciones con alcances tan diferentes a las de otro grupo, siendo que ambos comparten las mismas actitudes. Es decir, ¿qué estaría sustentando que las mismas actitudes llevaran a conductas diferentes? ¿Qué motivaría una acción colectiva y organizada de un grupo con representaciones sociales sobre el impacto de termoeléctricas en el medioambiente, versus acciones más aisladas y de corto alcance o microsociales, en otro grupo? Lo anterior, considerando la similitud de las representaciones sociales en ambos grupos.

La respuesta a éstas y otras interrogantes cobrarían gran importa en aquellas futuras investigaciones en las que la Psicología quisiera intervenir con el fin de dar cuenta del papel de los componentes intersubjetivos en las decisiones de organización social en grupos indignados con el impacto de termoeléctricas. Este estudio deja en evidencia que aunque las representaciones sociales puedan ser compartidas, y comunes en diferentes grupos u organizaciones, no siempre consiguen explicar las actuaciones que derivan de las interpretaciones del mundo. Existe un desafío para la Psicología Social en este sentido, toda vez que aparentemente no son sólo las representaciones compartidas, o grupos de creencias coletivas, las que logran explicar la articulación de acciones comunes, existiendo posibilidades de acción que posiblemente se encuentren vinculadas con aspectos históricos, o sociales, que finalmente gatillan la conducta luego incluso de una problematización común.

\section{Referencias}

Abric, J. (2001). Las representaciones sociales: aspectos teóricos. En J. Abric (Comp.), Prácticas Sociales y Representaciones (pp. 11-32). México: Ediciones Coyoacán

Alvira, F., García, M. \& Ibáñez, J. (2003). El análisis de la realidad social: Métodos y técnicas de investigación. Madrid: Alianza.

Aparicio, M. \& Mazzitelli, C. (2010). El abordaje del conocimiento cotidiano desde la teoría de las representaciones sociales. Revista Eureka sobre Enseñanza y Divulgación de las Ciencias, 7(3), 636-652. doi:10498/9816

Araya, S. (2004). Las representaciones sociales: Ejes teóricos para su discusión. Costa Rica: FLACSO.

Arancibia, S., Rodríguez, G., Fritis, R., Tenorio, N. \& Poblete, H. (2013). Representaciones Sociales en Torno a Equidad, Acceso y Adaptación en Educación Universitaria. Psicopers- pectivas, 12(1), 116-138. doi:10.5027/psicoperspectivas-Vol12Issue 1-fulltext-236

Bangher, M. (2005). Dignidad, Calidad de Vida y Derechos Humanos. Revista Estudios en Ciencias Humanas, 2. Recuperado de http:// hum.unne.edu.ar/revistas/postgrado/revista2/10_bangher.pdf

Besnier, D. (2010). Estudio narrativo sobre la construcción de significados en torno a la identidad social de los pescadores artesanales de una bahía de la $V$ región (Tesis de licenciatura inédita). Universidad del Mar, Chile.

Bravo, C. (2010). Hacia una narrativa de la naturaleza: la psicología ante el reto sustentable. Polis, Revista Latinoamericana, 9(26), 223-231. doi:10.4067/S0718-65682010000200011

Calixto, R. (2008). Representaciones sociales del medio ambiente. Perfiles educativos, 30(120), 33-62. Recuperado de http://www.scielo.org.mx/scielo.php?script=sci_arttext\&pid $=$ S0185-26982008000200003

Canales, M. (2006) Metodologías de Investigación Social: Introducción a los Oficios. Santiago: LOM Ediciones.

Cárcamo, P., Cortés, M., Ortega, L., Squeo, F. \& Gaymer, C. (2011). Crónica de un conflicto anunciado: Tres centrales termoeléctricas a carbón en un hotspot de biodiversidad de importancia mundial. Revista Chilena de Historia Natural, 84(2), 171-180. doi:10.4067/ S0716-078X2011000200003

Castillo, J. \& Winkler, M. (2010). Praxis y Ética en Psicología Comunitaria: Representaciones Sociales de Usuarias y Usuarios de Programas Comunitarios en la Región Metropolitana. Psykhe (Santiago), 19(1), 31-46. doi:10.4067/S0718-22282010000100003

Centro de Análisis de Políticas Públicas (2010). Informe país. Estado del medio ambiente en Chile 2008. Chile: Centro de Análisis de Políticas Públicas, Universidad de Chile. Recuperado de http:// www.uchile.cl/publicaciones/64137/informe-pais-estado-delmedio-ambiente-en-chile-2008

Contreras, C., Ibarra, C. \& Ulloa, P. (2011). Programa para el control de las emisiones al aire del sector industrial 2010-2014. Chile: Ministerio del Medio Ambiente, división políticas y regulaciones. Recuperado de http://www.sinia.cl/1292/articles-49901_Industrias.pdf

De la Torre, A. (2010). Estudio descriptivo de los significados presente en la construcción de identidad social de integrantes de movimientos ecologistas/ambientalistas de la bahía de Quintero (Tesis de licenciatura inédita). Universidad del Mar, Chile.

El desafío medioambiental pendiente (2011). Revista Electricidad Interamericana. Recuperado de http://www.revistaei.cl/reportajes/ el-desafio-medioambiental-pendiente/

García, E. (1999). Derechos Humanos y calidad de vida. En R. Gonzalez \& G. Arnaiz (Coord.), Derechos humanos: la condición humana en la sociedad tecnológica (pp. 131-163). Madrid: Tecnos.

González, I., Muena, V., Cisternas, M. \& Neaman, A. (2008). Acumulación de cobre en una comunidad vegetal afectada por contaminación minera en el valle de Puchuncaví, Chile central. Revista Chilena de Historia Natural, 81(2), 279-291. doi:10.4067/ S0716-078X2008000200010

Hernández, R., Fernández, C. \& Baptista, P. (2010). Metodología de la investigación. México: Mc Graw Hill.

Instituto Nacional de Estadísticas (2010). Compendio Estadístico Año 2010. Recuperado de http://www.ine.cl/canales/menu/publicaciones/compendio_estadistico/compendio_estadistico2010.php

Jodelet, D. (1986). La representación social: fenómeno, concepto y teoría. En S. Moscovici (Comp.), Psicología Social II: Pensamiento y vida social. Psicología social y problemas sociales (pp. 469-494). Barcelona: Paidós.

Barría, C. (2009). Análisis general del impacto económico y social de una norma de emisión para termoeléctricas. (Informe final preparado para la Comisión Nacional del Medioambiente, 
Gobierno de Chile). Recuperado de http://www.sinia.cl/1292/ articles-44963_informe_final_term.pdf

Kohn, M. (1976). Looking back. A 25-year review and appraisal of social problems research. Social Problems, 24(1), 94-112. doi: $10.2307 / 800325$

Mora, M. (2002). La teoría de las representaciones sociales de Serge Moscovici. México: Universidad de Guadalajara.

Moscovici, S. (1979). El psicoanálisis, su imagen y su público. Buenos Aires: Huemul.

Páez, D. (1987). Pensamiento, Individuo y Sociedad: cognición y representación social. Madrid: Fundamentos.

Pérez Serrano, G. (1994). Investigación Cualitativa. España: Editorial La Muralla.

Programa Chile Sustentable (2004). Crisis energética en Chile: rol y futuro de las energías renovables no convencionales. Recuperado de http://www.archivochile.com/Chile_actual/patag_sin_repre/03/ chact_hidroay-3\%2000025.pdf

Quintana, A. (2006). Metodología de Investigación Científica Cualitativa. En A. Quintana \& W. Montgomery (Eds.), Psicología: Tópicos de Actualidad (pp. 47-84). Lima: Universidad Nacional Mayor de San Marcos.

Román, R. \& Hall, S. (2011). El Futuro Energético de Chile está en la Eficiencia Energética y las Energías Renovables. Recuperado de http://www.futurorenovable.cl/wp-content/uploads/downloads/2011/07/Futuro-con-Renovables-y-Eficiencia-Energetica.pdf

Ruiz Olabuénaga, J. (1996). Metodología de la investigación cualitativa. Bilbao: Universidad de Deusto.
Salamanca, A. \& Martín-Crespo, C. (2007a). El diseño en la investigación cualitativa. Nure Investigación, 26. Recuperado de http:// www.nureinvestigacion.es/FICHEROS_ADMINISTRADOR/F_ METODOLOGICA/FMetodologica_26.pdf

Salamanca, A. \& Martín-Crespo, C. (2007b). El Muestreo En La Investigación Cualitativa. Nure Investigación, 27. Recuperado de http:// www.nureinvestigacion.es/FICHEROS_ADMINISTRADOR/F_ METODOLOGICA/FMetodologica_27.pdf

Superintendencia del Medio Ambiente (2014). Guía de aspectos ambientales relevantes para centrales termoeléctricas. Chile: Superintendencia del Medio Ambiente.

Tójar, J. \& Serrano, J. (2000). Ética e investigación educativa. Revista Electrónica de Investigación y Evaluación Educativa, 6(2). Recuperado de http://www.uv.es/RELIEVE/v6n2/RELIEVEv6n2 2.htm

Vega, M., Lyon, J. \& Robles, M. (3 de abril de 2011). La grave contaminación de Ventanas que la normativa chilena permite y que podría extenderse. Diario El Mercurio Domingo. Recuperado de http://diario.elmercurio.com/2011/04/03/reportajes/_porta$\mathrm{da} /$ noticias/88525A82-D366-405A-AE8F-5C18A24424DE. $\mathrm{htm} ? \mathrm{id}=\% 7 \mathrm{~b} 88525 \mathrm{~A} 82-\mathrm{D} 366-405 \mathrm{~A}-\mathrm{AE} 8 \mathrm{~F}-5 \mathrm{C} 18 \mathrm{~A} 24424 \mathrm{DE} \% 7 \mathrm{~d}$

Vera, M. (2007). Significado de la calidad de vida del adulto mayor para sí mismo y para su familia. Anales de la Facultad de Medicina, 68(3), 284-290. doi:10.15381/anales.v68i3.1218

Wiesenfeld, E. (2003). La Psicología ambiental y el desarrollo sostenible ¿Cuál Psicología ambiental? ¿Cuál desarrollo sostenible?. Estudos de Psicologia (Natal), 8(2), 253-261. doi:10.1590/ S1413-294X2003000200007 
